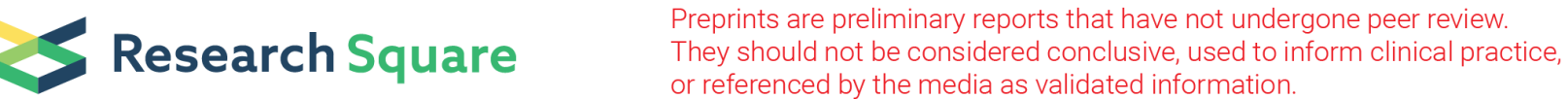

\section{NDRG2 Inhibits Pyruvate Carboxylase-Mediated Anaplerosis and Combines with Glutamine Blockade To Inhibit The Growth of Glioma}

\section{Jiancai Wang}

Fourth Military Medical University Bethune Military Medical College: Army Medical University

\section{Xiang Sun}

Fourth Military Medical University Bethune Military Medical College: Army Medical University Jiayuan Wang

Fourth Military Medical University Bethune Military Medical College: Army Medical University

Kun Zhang

Fourth Military Medical University: Air Force Medical University

\section{Yiyi Yuan}

Fourth Military Medical University Bethune Military Medical College: Army Medical University Libo Yao

Fourth Military Medical University Bethune Military Medical College: Army Medical University

\section{Xia Li}

Fourth Military Medical University Bethune Military Medical College: Army Medical University

Lan Shen ( $\square$ lanshen@fmmu.edu.cn)

Fourth Military Medical University

\section{Research}

Keywords: NDRG2, anaplerosis, puruvate carboxylase, glioma

Posted Date: September 21st, 2021

DOI: https://doi.org/10.21203/rs.3.rs-892486/v1

License: (1) (1) This work is licensed under a Creative Commons Attribution 4.0 International License. Read Full License 


\section{Abstract \\ Background}

Due to the rapid proliferation, cancer cells have increased anabolic biosynthesis, which requires anaplerosis to replenish precursor intermediates. The major anaplerotic sources are pyruvate and glutamine, which require the catalysis of pyruvate carboxylase (PC) and glutaminase (GLS) respectively. In GLS-suppressed cancer cells, the PC-mediated pathway for anaplerosis is crucial to maintain cell growth and proliferation. Here, we investigated the regulatory role and molecular mechanism of the tumor suppressor NDRG2 in PC and PC-mediated anaplerosis.

\section{Methods}

We first evaluated the correlation between PC and NDRG2 in glioma cell lines and human glioma tissue microarrays. The interaction between PC and NDRG2 was tested by tandem affinity purification-mass spectrometry (TAP-MS) and coimmunoprecipitation (Co-IP). We then detected the effect of NDRG2 on PCmediated anaplerosis by gas chromatography-mass spectrometry (GC-MS). Preclinical evaluation of NDRG2 alone or combined with a glutaminase inhibitor was conducted in glioma cells and xenograft mouse tumors.

\section{Results}

NDRG2 interacted with PC and induced the degradation of PC in glutamine-deficient glioma cells. NDRG2 also inhibited the activity of PC and PC-mediated anaplerosis. As a result, NDRG2 significantly inhibited the malignant growth and proliferation of glioma cells in combination with a glutamine antagonist. In addition, NDRG2 more significantly inhibited the protein level of PC in IDH1(R132H)-mutant glioma cells than in wild-type glioma cells.

\section{Conclusions}

These findings indicate that the molecular mechanism of NDRG2 inhibits PC-mediated anaplerosis and collaborates with glutamine antagonist to inhibit the malignant proliferation of glioma cells, thus providing a theoretical and experimental basis for targeting anaplerosis in glioma therapy.

\section{Background}

Malignant growth and proliferation of cancer cells require large quantities of bioenergy and biomaterials [1]. Many biosynthetic precursors for proteins, lipids and nucleotides are generated from the tricarboxylic acid (TCA) cycle. When the intermediates are removed from the TCA cycle, additional metabolic pathways are necessary to supply oxaloacetate (OAA) and TCA cycle intermediates. These OAA- 
generating metabolic pathways are named anaplerosis, which is used to replenish precursor intermediates [2,3]. The major anaplerotic resources are pyruvate and glutamine, which require the enzymatic activity of pyruvate carboxylase (PC) and glutaminase (GLS), respectively [4]. When glutamine is deprived, PC-mediated anaplerosis is important for the growth and proliferation of cancer cells.

Gliomas are the most common and the most malignant brain tumors. The World Health Organization divides gliomas into four grades [5]. Grade I gliomas are benign, grades II and III are diffuse gliomas that are invasive and can progress into grade IV, which is also named glioblastoma [6]. Somatic mutations in the isocitrate dehydrogenase 1 gene (IDH1) occur at a very high frequency in grade II/III gliomas and secondary glioblastomas, and IDH1 mutations seem to be a prognostic factor for survival in glioma patients $[7,8]$. IDH1 mutations, which commonly occur at the R132 residue in the active site, lead to the production of the oncometabolite $2 \mathrm{HG}$ and tumorigenesis [9]. Furthermore, IDH1 mutations elicit additional metabolic changes, especially in reprogramming of pyruvate metabolism [9]. Pyruvate dehydrogenase $(\mathrm{PDH})$ activity is decreased while pyruvate carboxylase $(\mathrm{PC})$ activity and expression are increased in IDH1-mutated glioma cells, which results in the enhancement of PC-mediated TCA anaplerosis $[9,10]$. PC and PC-mediated TCA anaplerosis may be potential targets in IDH1-mutated gliomas.

$\mathrm{N}$-myc downstream-regulated gene 2 (NDRG2), a member of the NDRG family, was firstly discovered by our laboratory using subtractive hybridization [11]. Our previous studies have demonstrated that NDRG2 is expressed widely in normal tissues [12], and is decreased in glioblastoma and other types of tumor tissues [11, 13-16]. NDRG2 can inhibit the malignant growth and proliferation of tumor cells $[11,17,18]$, and is therefore considered a tumor suppressor gene $[19,20]$. Moreover, NDRG2 suppresses tumor metabolic reprogramming. Our previous research showed that NDRG2 inhibited glycolysis and glutaminolysis by coordinately targeting glucose and glutamine transporters, multiple catalytic enzymes involved in glycolysis and glutaminolysis of cancer cells [1]. NDRG2 also inhibited the activation of fatty acid oxidation and suppressed the glucose deprivation-induced AMPK/ACC pathway activation in cancer cells [21]. As a tumor suppressor gene, NDRG2 plays important roles in tumor metabolic reprogramming.

In this study, we aimed to investigate the regulatory role of NDRG2 in pyruvate carboxylase and pyruvate carboxylase-mediated anaplerosis. We found that NDRG2 interacted with PC and induced the degradation of PC, thus inhibiting the activity of PC and PC-mediated anaplerosis. Moreover, NDRG2 combined with a GLS inhibitor significantly inhibited the malignant growth and proliferation of glioma cells. Our findings may provide potential molecular targets for glioma therapy.

\section{Methods}

\section{Cell culture and materials}

The human glioma cell lines U251, T98G, IDH1(R132H) U87, IDH1(WT) U87 and HEK-293T cells were purchased from ATCC and used in the present study. Cells were maintained in the medium recommended 
and supplemented with $10 \% \mathrm{FBS}$ in a $37^{\circ} \mathrm{C}$ and $5 \% \mathrm{CO}_{2}$ incubator. Recombinant lentiviral vectors were constructed with an Invitrogen ViraPower ${ }^{\mathrm{TM}}$ Lentiviral System (Carlsbad) in our laboratory.[1] The lentiviral vectors pLenti6-mCherry/NDRG2, PAX2 and PMD2G were transfected into HEK-293T cells using Lipofectamine 2000 (Invitrogen) according to the manufacturer's instructions. Glioma cells were infected with viral medium from HEK-293T cells $48 \mathrm{~h}$ after transfection.

\section{MTT assay}

Infected cells were seeded in 96-well plates in triplicate at a starting density of $1 \times 10^{4}$ cells $/$ well and cultured in the recommended medium without glutamine. Treated cells were washed and incubated with tetrazolium salt (MTT, $100 \mu \mathrm{g} / \mathrm{ml}$; Sigma) at $37^{\circ} \mathrm{C}$ for $4 \mathrm{~h}$. The supernatant was removed, and $150 \mu \mathrm{l}$ of dimethyl sulfoxide (DMSO) was added to each well. The absorbance (OD) of the reaction solution at 490 $\mathrm{nm}$ was recorded.

\section{Colony formation assay}

Infected cells were seeded into $60-\mathrm{mm}$ dishes at a density of 400 cells per dish. The cells were grown for 2 weeks in culture medium without glutamine. Then, the colonies were fixed and stained with crystal violet.

\section{Western blotting analysis}

For Western blotting analysis, total protein was prepared from human liver cell lines and clinical hepatocellular carcinoma tissue samples. Immunoblotting was performed according to standard procedures with monoclonal rabbit anti-PC antibody (Abcam ab126707, 1:2000), monoclonal mouse antiNDRG2 antibody (Abnova H00057447-M03, 1:1000), monoclonal mouse anti-Flag antibody (Sigma F3165, 1:1000), monoclonal mouse anti-Myc tag antibody (Abcam ab32, 1:1000), monoclonal mouse anti-HA tag antibody (Abcam ab18181, 1:1000), monoclonal mouse anti-IDH1(R132H) antibody (Sigma ASB4200548, 1:200), monoclonal mouse anti-a-tubulin antibody (Boster M03989-2, 1:1000) and monoclonal rabbit anti- $\beta$-actin antibody (Boster BM3873, 1:1000).

\section{Quantitative real-time PCR}

Total RNA was isolated from cells using TRIzol Reagent (Invitrogen), and then complementary DNA (CDNA) was synthesized using AMV reverse transcriptase (Promega) according to the manufacturer's instructions. cDNA was used as a template for quantitative real-time PCR using an ABI Prism 7500 realtime PCR instrument (Applied Biosystems). The primers used for real-time quantitative PCR are listed in Table S1.

\section{Vector construction}

The construction scheme of the expression vector used for tandem affinity purification (TAP) is shown in Fig. 3A. For construction of the expression vector, the coding genes of NDRG2 were amplified by PCR and confirmed by DNA sequencing, and then target genes were ligated with a fusion expression vector 
containing S-tag, Flag and streptavidin-binging peptide (SBP). The primers used for PCR are listed in Table S2.

\section{Tandem affinity purification}

HEK293T cells were transfected with an SFB-tagged NDRG2 or empty vector. Twenty-four hours posttransfection, the cells were lysed in NETN buffer $(20 \mathrm{mmol} / \mathrm{L} \mathrm{Tris-HCl}, \mathrm{pH} 8.0,100 \mathrm{mmol} / \mathrm{L} \mathrm{NaCl}, 1$ $\mathrm{mmol} / \mathrm{L}$ EDTA, $0.5 \%$ Nonidet P-40, $50 \mathrm{mmol} / \mathrm{L}$ b-glycerophosphate, $10 \mathrm{mmol} / \mathrm{L} \mathrm{NaF}$, and $1 \mathrm{mg} / \mathrm{mL}$ pepstatin A) at $4^{\circ} \mathrm{C}$ for 3 hours. The supernatant was collected for incubation with streptavidin Sepharose beads (GE Healthcare Sciences) at $4^{\circ} \mathrm{C}$ overnight. The next day, the beads were washed with NETN buffer five times and then eluted with $2 \mathrm{mmol} / \mathrm{L}$ biotin (Sigma) for 1 hour at $4^{\circ} \mathrm{C}$ twice. The elution products were incubated with $\mathrm{S}$ protein agarose beads (Novagen) at $4^{\circ} \mathrm{C}$ overnight, and after three washes, the products bound to S-protein agarose beads were subjected to SDS-PAGE and analyzed by mass spectrometry (MS).

\section{Coimmunoprecipitation}

Cells were harvested and lysed in IP buffer $(50 \mathrm{mmol} / \mathrm{L} \mathrm{Tris-HCl}, \mathrm{pH} 7.4,150 \mathrm{mmol} / \mathrm{L} \mathrm{NaCl}, 1 \%$ Triton X$100,1 \%$ sodium deoxycholate, and $1 \%$ protease inhibitor cocktails) on ice for more than 15 minutes. Cell lysates were centrifuged for 10 minutes at $13,000 \mathrm{rpm}$ at $4^{\circ} \mathrm{C}$, and the supernatant was transferred to a new tube. The supernatant was incubated with primary antibodies against Myc or Flag and protein A/G agarose beads (Thermo Fisher Scientific) with gentle rocking at $4^{\circ} \mathrm{C}$ overnight. The next day, the pellet was washed six times with IP buffer on ice and then subjected to Western blotting analysis.

\section{Immunohistochemistry}

Glioma specimens were histologically diagnosed, and glioma tissue microarrays (TMAs) were produced by the Department of Pathology, Xijing Hospital, Fourth Military Medical University. Tissue microarray (TMA) staining was performed using standard immunohistochemistry procedures. The slides were incubated overnight with primary antibodies against NDRG2 (Abnova H00057447-M03, 1:500) or PC (Abcam ab229267, 1:500). Staining intensity was scored in a blinded fashion: $1=$ weak staining at $\times 100$ magnification but little or no staining at $\times 40$ magnification; $2=$ medium staining at $\times 40$ magnification; 3 $=$ strong staining at $\times 40$ magnification. The final staining index was calculated using the following formula: staining intensity $\times$ percentage.

Tumor tissues from nude mice were collected on day 28 , excised and fixed with $4 \%$ formalin, and embedded in paraffin. For immunohistochemistry, $5 \mu \mathrm{m}$-thick tissue sections were cut, dewaxed in xylene, and rehydrated. For Ki67 staining, the slides were incubated with $1 \%$ bovine serum albumin in PBS at room temperature for $1 \mathrm{~h}$ for blocking and then stained with primary antibodies against NDRG2 (Abnova H00057447-M03, 1:500), PC (Abcam ab229267, 1:500) or Ki-67 (Invitrogen, PA5-19462, 1:1000) at room temperature for $4 \mathrm{~h}$. The slides were subsequently washed three times with PBS to remove excess primary antibody and then incubated with anti-mouse HRP-conjugated IgG (Boster BM3895, 1:500) for 1 $\mathrm{h}$ at room temperature. Finally, the slides were washed three times, incubated with DAB peroxidase 
substrate (Sigma) and covered with glass cover slips. The staining results were observed with a bright field microscope.

For NDRG2 and PC subcellular localization, the cells were fixed in a freshly prepared solution of $4 \%$ paraformaldehyde, rinsed, and permeabilized with $0.1 \%$ Triton X-100 in PBS. Permeabilized cells were then incubated with horse serum in PBS to block nonspecific binding. After thorough rinsing with PBS, the cells were incubated overnight with NDRG2 or PC antibody, and incubated with fluorescein isothiocyanate (FITC)-conjugated anti-rabbit antibody or Cy5-conjugated anti-mouse antibody. Dual-color detection was performed by confocal laser scanning microscopy after treatment with 4',6-diamidino-2-phenylindole (DAPI) for 10 min to label nuclear DNA.

\section{Pyruvate carboxylase activity}

Treated cells were seeded on 6-well plates at a density of $1 \times 10^{6}$ cells per well and the culture medium was changed to low glucose DMEM without phenol red (Thermo Fisher Scientific). The activity of pyruvate carboxylase in the culture medium was measured after incubation of cells for $24 \mathrm{~h}$ with a pyruvate carboxylase activity assay kit (Jiancheng Bioengineering). The activity of pyruvate carboxylase was normalized to the cell numbers. The cell numbers were calculated and analyzed using a Cellometer Mini bright field automated cell counter (Nexcelom Bioscience).

\section{Tracer studies in cell cultures}

U251 cells transduced with NDRG2 or mCherry were incubated in DMEM with $10 \mathrm{mM}{ }^{13} \mathrm{C}$-glucose and in the absence of glutamine for 18 hours, quenched in cold acetonitrile, and extracted in acetonitrile/water/chloroform ( $\mathrm{v} / \mathrm{v}$ 2:1.5:1). Metabolite fractions from cells were analyzed by mass spectrometry (MS) as previously reported [22].

\section{In vivo tumorigenicity assay}

The animal study and experimental protocols were approved by the Institutional Laboratory Animal Center at the Fourth Military Medical University. The animals were maintained and handled in accordance with the Guidelines for Accommodation and Care of Animals. JC Wang has a license for animal experiments. All mice were housed under standard conditions of a 12-hour light/dark cycle and access to food and water ad libitum. Four-week-old athymic mice were injected subcutaneously with $1 \times 10^{6}$ cells. U251 cells expressing the mCherry control were injected into the left flank, and U251 cells expressing NDRG2 were injected into the right flank. When the tumor size reached an average of $60 \mathrm{~mm}^{3}$, the mice were treated with $15 \mathrm{mg} / \mathrm{kg} \mathrm{L-DON}$ or PBS 3 times per week. The tumor size was measured every other day, and tumor volume was calculated using a standard formula: tumor volume $\left(\mathrm{mm}^{3}\right)=$ width $\left(\mathrm{mm}^{2}\right) \times$ length $(\mathrm{mm}) \times 0.5$. Eleven days after drug treatment, the mice were sacrificed and the tumors were harvested for Western blot analysis.

\section{Statistical analysis}


Statistical analysis was performed with SPSS software (version 17.0; SPSS). The results are presented as the mean \pm SEM from at least three individual experiments for each group. Student's t-test was used to compare the differences between two groups. Pearson product-moment correlation was used to calculate the correlation between NDRG2 and PC staining index in IDH1 wild-type and IDH1-R132H mutant glioma TMAs. Statistical significance was defined as $p<0.05$, and statistical graphics were prepared with Origin 6.0 (Microcal Software, Inc., Northampton).

\section{Results}

NDRG2 inhibits cell growth and blocks the upregulation of pyruvate carboxylase expression in glutaminedeficient glioma cells

To investigate the effect of NDRG2 on glutamine-deficient glioma cells, we initially examined the growth and proliferation of NDRG2-overexpressing glioma cells in the glutamine-deficient culture medium by MTT and colony formation assays. Interestingly, ectopic NDRG2 expression markedly diminished the growth and proliferation of U251 and T98G cells in the glutamine-deficient culture conditions (Fig. 1A and B). Under glutamine deprivation, PC-mediated anaplerosis is important for the growth and proliferation of cancer cells. Therefore, we investigated the regulatory effect of NDRG2 on the expression of pyruvate carboxylase in the absence of glutamine metabolism. We observed the transcription and protein levels of pyruvate carboxylase in glutamine-deficient glioma cells with ectopic NDRG2 expression. Immunoblot analysis showed that the expression of pyruvate carboxylase increased under glutamine deprivation in the glioma cell lines U251 and T98G. However, the expression of pyruvate carboxylase decreased slightly in the NDRG2 -overexpressing U251 and T98G cells (Fig. 1C). Interestingly, the mRNA level of pyruvate carboxylase increased under glutamine deprivation in glioma cells (Fig. S1). NDRG2 blocked the upregulation of pyruvate carboxylase expression induced by glutamine deprivation in glioma cells at the protein level. These results suggest that pyruvate carboxylase may be regulated post-translationally by NDRG2 in glutamine-deficient cells.

\section{NDRG2 induces the ubiquitination and degradation of pyruvate carboxylase under glutamine deficiency}

As NDRG2 blocked the upregulation of pyruvate carboxylase expression induced by glutamine deprivation only at the protein level, we hypothesized that NDRG2 might regulate the protein stability and degradation of pyruvate carboxylase. To test this hypothesis, we infected the glioma cell lines U251 and T98G with lentivirus containing NDRG2 or mCherry, and then treated them with cycloheximide (CHX), a protein synthesis inhibitor. The results of immunoblot analysis showed that the protein levels of pyruvate carboxylase decreased more significantly in the NDRG2-overexpressing glioma cells treated with cycloheximide, than in the control cells (Fig. 2A). The degradation of pyruvate carboxylase was significantly accelerated by NDRG2 overexpression in the presence of CHX, suggesting that NDRG2 could promote the protein degradation of pyruvate carboxylase. 
Ubiquitinated modification of proteins is usually involved in proteasome degradation [23]. We first examined whether the protein degradation of pyruvate carboxylase depends on the proteasome. As shown in Fig. 2B, NDRG2 inhibited the protein level of pyruvate carboxylase, but the proteasome inhibitor MG132 blocked the decrease of pyruvate carboxylase in the NDRG2-overexpressing U251 and T98G glioma cells. Second, we examined whether NDRG2 affects the ubiquitination of pyruvate carboxylase. As shown in Fig. 2C, overexpression of NDRG2 significantly promoted the ubiquitination of pyruvate carboxylase under glutamine deficiency. Therefore, NDRG2 induces the ubiquitination and degradation of pyruvate carboxylase under glutamine deficiency.

\section{NDRG2 interacts with pyruvate carboxylase}

Since NDRG2 regulates the ubiquitination and degradation of pyruvate carboxylase, we wondered whether an interaction exists between NDRG2 and pyruvate carboxylase. Tandem affinity purification (TAP) and mass spectrometry assays were used to search for the interacting proteins of NDRG2. We first constructed a NDRG2 mammalian expression vector, which contains S, Flag, and streptavidin-bindingpeptide tags (Fig. 3A). The cDNA coding fragment of NDRG2 was amplified from human brain mRNA by RT-PCR (Fig. 3B) and cloned into the pMD18-T vector to analyze the nucleotide sequence. The results showed that the DNA sequence of the cloned human NDRG2 domain was consistent with that reported previously [11]. The NDRG2 cDNA was subcloned into the expression vector, and the recombinant plasmid was digested with restriction enzymes (Fig. 3C). The recombinant plasmid and the backbone vector were individually transfected into HEK293T cells, and the expression of NDRG2 fusion protein was detected by Western blots (Fig. 3D). Then, HEK293T cell extracts were prepared for tandem affinity protein purification to obtain the NDRG2 protein complex, which was then detected by mass spectrometry. Multiple binding partners of NDRG2, including pyruvate carboxylase, were identified by mass spectrum (Fig. 3E and Table S3).

NDRG2 is mainly located in the cytoplasm of astrocytes,[12, 13] and pyruvate carboxylase is mainly located in the mitochondria [24]. Therefore, we detected the colocalization and interaction of NDRG2 and pyruvate carboxylase in glioma cells. Our indirect immunofluorescence results showed that NDRG2 and pyruvate carboxylase were well colocalized in U251 cells (Fig. 3F and Fig. S2). Moreover, the interaction between ectopically expressed Flag-NDRG2 and Myc-PC in HEK293T cells and endogenous NDRG2 and PC in U251 cells was confirmed by reciprocal coimmunoprecipitation (co-IP) assays (Fig. 3G).

\section{NDRG2 inhibits the activity of pyruvate carboxylase and glucose-dependent anaplerosis through pyruvate carboxylase in glioma cells}

Pyruvate carboxylase mediates glucose-dependent anaplerosis [3], we characterized the regulatory effect of NDRG2 on pyruvate carboxylase activity and anaplerosis mediated by pyruvate carboxylase. Our results showed that NDRG2 inhibited the activity of PC in U251 and T98G glioma cells. In the NDRG2 overexpressing U251 and T98G glioma cells, the activity of pyruvate carboxylase decreased (Fig. 4A). To verify the inhibitory effect of NDRG2 on PC-mediated anaplerosis in cells, we incubated U251 cells that were infected with lentivirus containing NDRG2 or mCherry in ${ }^{13} \mathrm{C}$-glucose for 24 hours and measured the 
metabolites for PC-mediated anaplerosis by GC-MS. The levels of ${ }^{13} \mathrm{C}$-oxaloacetate, ${ }^{13} \mathrm{C}$-citrate, ${ }^{13} \mathrm{C}$ malate and ${ }^{13} \mathrm{C}$-succinate were reduced in the NDRG2 overexpressing U251 cells compared with the control cells (Fig. 4B). Therefore, NDRG2 inhibited PC-mediated anaplerosis in glioma cells.

\section{NDRG2 combined with a glutamine antagonist predominantly inhibits the proliferation of glioma cells}

Pyruvate carboxylase-mediated anaplerosis is required for the growth and proliferation of glioma cells [3]. Accordingly, we detected the effect of NDRG2 combined with a glutamine antagonist on the growth and proliferation of glioma cells in vivo. We established xenograft tumors using U251 cells overexpressing either mCherry or NDRG2. Once tumors were established, mice were treated with the glutaminase inhibitor L-DON for 21 days (Fig. 5A). Importantly, the mice injected with U251 cells expressing NDRG2 and treated with L-DON developed tumors more slowly that the mice in the other groups (Fig. 5B-D). In the related tumor sections, the protein levels of the proliferation marker Ki-67 and PC decreased significantly after NDRG2 overexpression and L-DON treatment (Fig. 5E). Thus, NDRG2 combined with a glutamine antagonist can inhibit the malignant growth and proliferation of glioma cells.

\section{The expression of NDRG2 has an inverse association with pyruvate carboxylase in IDH1(R132H)-mutant glioma cells}

Pyruvate carboxylase serves as a major source of TCA anaplerosis, while glutamine is used for 2hydrozyglutarate production in IDH1 mutant glioma cells [10]. To fully address the clinical relevance of NDRG2 and pyruvate carboxylase in IDH1-R132H mutant glioma patients, we detected the protein levels of NDRG2 and pyruvate carboxylase in IDH1 wild type and IDH1-R132H mutant glioma tissues from 54 patients. Immunohistochemical and statistical analyses showed that there was no significant association between NDRG2 and pyruvate carboxylase expression in the IDH1 wild-type glioma tissues, but there was an inverse association between NDRG2 and pyruvate carboxylase expression in the IDH1R132H mutant glioma tissues (Fig. 6A and B). We found that the expression of pyruvate carboxylase was upregulated in the IDH1-R132H mutant U87 glioma cells. However, ectopic NDRG2 expression decreased pyruvate carboxylase expression levels in the IDH1-R132H mutant U87 glioma cells (Fig. 6C and D). NDRG2 may inhibit the protein level of pyruvate carboxylase in the IDH1 mutant glioma cells.

In summary, this study illustrates the regulatory role and molecular mechanism of the tumor suppressor NDRG2 in PC and PC-mediated anaplerosis in glioma cells. In glucose homeostasis glia cells, NDRG2 induces the degradation of partial PC via ubiquitin proteasome pathway, and thereby partly inhibiting PCmediated anaplerosis upon glutamine deprivation in mitochondria. In glioma cells, NDRG2 loss enhanced PC and PC-mediated anaplerosis, which facilitated the malignant proliferation of glioma cells (Fig. 6E). In addition, PC expression was upregulated in the IDH1-R132H mutant U87 glioma cells compared with the IDH1 wild-type U87 glioma cells. NDRG2 inhibited the upregulation of PC expression in the IDH1 mutant U87 glioma cells. The inhibition of anaplerosis may be one of the important reasons for NDRG2 as a tumor suppressor. Therefore, tumor suppressor NDRG2 is a potential therapeutic target for glioma. 


\section{Discussion}

Tumor cells use glucose and glutamine to fuel anaplerosis and cell proliferation, which are catalyzed by pyruvate carboxylase and glutaminase respectively. Pyruvate carboxylase (PC) is the key anaplerotic enzyme that converts pyruvate to oxaloacetate in mitochondria, enabling the maintenance of other metabolic intermediates consumed by cataplerosis [25]. In glutamine-blocked tumor cells, the induction of a compensatory anaplerotic mechanism catalyzed by PC, allowed the cells to use glucose-derived pyruvate rather than glutamine for anaplerosis [3]. Glutamine blockade is widely used in cancer targeted therapy and immunotherapy, but resistance to glutamine blockade poses considerable challenges to cancer therapies. Therefore, the use of combination therapy is an effective strategy in the treatment of certain types of cancers [26].

Pyruvate carboxylase expression is upregulated in many types of cancer, including glioma. The promoter of the human PC gene contains the binding sites of several transcription factors, such as C-Myc, HIF1, SP1 and the vitamin D-responsive element (VDRE) [27]. These oncogenic transcription factors may contribute to the upregulation of PC gene expression in tumor cells. Glutamine blockade attenuates the production of biosynthesis and bioenergy, and therefore induces the inhibition of the mTOR signaling pathway and activation of AMPK $[28,29]$. In addition, glutamine blockade leads to upregulation of the transcriptional factor c-Myc and activation of transcription factor 4 [30-32]. Our results showed that glutamine blockade induced the upregulation of pyruvate carboxylase at the transcriptional level, which is probably related to the upregulation or activation of transcription factors such as c-Myc. Clarifying the mechanism by which glutamine blockade induces the upregulation of pyruvate carboxylase expression could reveal new therapeutic targets for anaplerosis in cancer.

Pyruvate carboxylase is mainly located in mitochondria, which catalyzes the carboxylation of pyruvate to form oxaloacetate and maintain TCA cycle flux during robust biosynthesis [3]. The degradation of pyruvate carboxylase in mitochondria, similar to many enzymes in mitochondria, mainly occurs through the autophagic-lysosomal degradation pathway [33,34]. Interestingly, our results showed that the tumor suppressor NDRG2 promoted the degradation of pyruvate carboxylase through the ubiquitin-proteasome degradation pathway in the cytoplasm, and maintained the remaining amount of pyruvate carboxylase entering the mitochondria.

Tumor suppressor gene NDRG2, which is mainly expressed in the cytoplasm, can inhibit tumor metabolic reprogramming and malignant growth. Our results showed that NDRG2 interacted with pyruvate carboxylase and induced the degradation of pyruvate carboxylase via the ubiquitin proteasome pathway. Moreover, NDRG2 inhibited PC-mediated anaplerosis and glioma cell proliferation upon glutamine deprivation. In addition, NDRG2 cooperated with a glutamine antagonist to suppress the malignant growth of glioma cells. It is possible that the tumor suppressor gene NDRG2 induces sensitivity to glutamine antagonism-targeted cancer therapies.

Gliomas are the most common primary malignancy of the central nervous system and are derived from supporting glia. Approximately $60-90 \%$ of low-grade gliomas and secondary glioblastomas harbor a 
heterozygous $\mathrm{R} 132 \mathrm{H}$ mutation in the gene coding for isocitrate dehydrogenase 1 (IDH1) [35]. Consistent with other research results, our results showed that the expression of PC increased significantly in the IDH1-mutant U87 cells compared with the wild-type IDH1 U87 cells [10]. Importantly, NDRG2 inhibited PC expression in the IDH1-mutant U87 cells compared with the wild-type IDH1 U87 cells. NDRG2 may be a potential molecular target for glioma therapy.

\section{Conclusions}

In this study, we provided the first evidence that NDRG2 interacts with pyruvate carboxylase and induces the degradation of pyruvate carboxylase in glutamine-blocked glioma cells. Therefore, NDRG2 inhibits PC-mediated and glucose-dependent anaplerosis in gliomas. Furthermore, NDRG2 more significantly inhibited the expression of PC in the IDH1 mutant gliomas than in the IDH1 wild-type gliomas. As a result, NDRG2 cooperated with glutamine inhibitor to significantly suppress the growth and proliferation of gliomas. NDRG2 might also be a potential therapeutic target for molecular targeted therapy of gliomas.

\section{Abbreviations}

NDRG2

N-myc downstream-regulated gene 2; PC:Pyruvate carboxylase; GLS:Glutaminase; TAP-MS:Tandem affinity purification-mass spectrometry; TCA:Tricarboxylic acid; Co-IP:Coimmunoprecipitation; DMSO:Dimethyl sulfoxide; IDH1:Isocitrate dehydrogenase 1; FITC:Fluorescein isothiocyanate; PDH:Pyruvate dehydrogenase; IHC:Immunohistochemstry; CHX:Cycloheximide; TMA:Tissue microarray; DAB:Diaminobenzidine tetrahydrochloride; VDRE:Vitamin D-responsive element

\section{Declarations}

\section{Ethics approval and consent to participate}

The study was conducted with the approval of the Ethics Committee of Xijing Hospital of The Fourth Military Medical University. All participating patients provided written informed consent. Nude mice used for in vivo experimental animal studies were cared for in accordance with a protocol approved by the Laboratory Animal Care and Use Committee of The Fourth Military Medical University.

\section{Consent for publication}

All contributing authors agree to the publication of this article.

\section{Availability of data and materials}

All data are fully available without restrictions.

\section{Competing interests}


The authors declare that they have no competing interests.

\section{Funding}

This work was supported by the National Natural Science Foundation of China (81672542 and 31171112), the Natural Science Foundation of Shaanxi Province (2021 JZ-27 and 2017SF-187), and the Foundation of Cancer Biology State Key Laboratory (No. CBSKL2019ZZ13).

\section{Authors' contributions}

LS designed and supervised the study. JCW, XS, JYW, KZ, and YYY collected and assembled the data. LS, LX and LBY provided administrative, technical, and material support. LS wrote and revised the manuscript. All authors read and approved the final manuscript.

\section{Acknowledgments}

We would like to thank our researchers for their hard work.

\section{Author details}

${ }^{1}$ The State Key Laboratory of Cancer Biology, Department of Biochemistry and Molecular Biology, The Fourth Military Medical University, Xi'an 710032, Shannxi,China. ${ }^{2}$ Department of neurosurgery, PLA 982 hospital, Tangshan 063099, Hebei, China. ${ }^{3}$ Department of Special Diagnosis, School of Stomatology, The Fourth Military Medical University, Xi'an, 710032, Shannxi,China. ${ }^{4}$ Department of Pathogenic Biology, Medical College, Yan 'an University, Yan 'an 716000 Shaanxi, China.

\section{References}

1. Xu X, Li J, Sun X, Guo Y, Chu D, Wei L, Li X, Yang G, Liu X, Yao L, et al. Tumor suppressor NDRG2 inhibits glycolysis and glutaminolysis in colorectal cancer cells by repressing c-Myc expression. Oncotarget. 2015;6(28):26161-76.

2. Owen $\mathrm{OE}$, Kalhan SC, Hanson RW. The key role of anaplerosis and cataplerosis for citric acid cycle function. J Biol Chem. 2002;277(34):30409-12.

3. Cheng T, Sudderth J, Yang C, Mullen AR, Jin ES, Mates JM, DeBerardinis RJ. Pyruvate carboxylase is required for glutamine-independent growth of tumor cells. Proc Natl Acad Sci U S A. 2011;108(21):8674-9.

4. Sellers K, Fox MP, Bousamra M 2nd, Slone SP, Higashi RM, Miller DM, Wang Y, Yan J, Yuneva MO, Deshpande R, et al. Pyruvate carboxylase is critical for non-small-cell lung cancer proliferation. J Clin Invest. 2015;125(2):687-98.

5. Louis DN, Ohgaki H, Wiestler OD, Cavenee WK, Burger PC, Jouvet A, Scheithauer BW, Kleihues P. The 2007 WHO classification of tumours of the central nervous system. Acta Neuropathol. 2007;114(2):97-109. 
6. Megova M, Drabek J, Koudelakova V, Trojanec R, Kalita O, Hajduch M. Isocitrate dehydrogenase 1 and 2 mutations in gliomas. J Neurosci Res. 2014;92(12):1611-20.

7. Weller $\mathrm{M}$, Wick W, von Deimling A. Isocitrate dehydrogenase mutations: a challenge to traditional views on the genesis and malignant progression of gliomas. Glia. 2011;59(8):1200-4.

8. Bleeker FE, Atai NA, Lamba S, Jonker A, Rijkeboer D, Bosch KS, Tigchelaar W, Troost D, Vandertop WP, Bardelli A, et al. The prognostic IDH1(R132) mutation is associated with reduced NADP+-dependent IDH activity in glioblastoma. Acta Neuropathol. 2010;119(4):487-94.

9. Izquierdo-Garcia JL, Viswanath P, Eriksson P, Cai L, Radoul M, Chaumeil MM, Blough M, Luchman HA, Weiss S, Cairncross JG, et al. IDH1 Mutation Induces Reprogramming of Pyruvate Metabolism. Cancer Res. 2015;75(15):2999-3009.

10. Izquierdo-Garcia JL, Cai LM, Chaumeil MM, Eriksson P, Robinson AE, Pieper RO, Phillips JJ, Ronen SM. Glioma cells with the IDH1 mutation modulate metabolic fractional flux through pyruvate carboxylase. PLoS One. 2014;9(9):e108289.

11. Deng Y, Yao L, Chau L, Ng SS, Peng Y, Liu X, Au WS, Wang J, Li F, Ji S, et al. N-Myc downstreamregulated gene 2 (NDRG2) inhibits glioblastoma cell proliferation. Int J Cancer. 2003;106(3):342-7.

12. Hu XL, Liu XP, Deng YC, Lin SX, Wu L, Zhang J, Wang LF, Wang XB, Li X, Shen L, et al. Expression analysis of the NDRG2 gene in mouse embryonic and adult tissues. Cell Tissue Res. 2006;325(1):67-76.

13. Shen L, Zhao ZY, Wang YZ, Ji SP, Liu XP, Liu XW, Che HL, Lin W, Li X, Zhang J, et al. Immunohistochemical detection of Ndrg2 in the mouse nervous system. Neuroreport. 2008;19(9):927-31.

14. Shen L, Qu X, Li H, Xu C, Wei M, Wang Q, Ru Y, Liu B, Xu Y, Li K, et al. NDRG2 facilitates colorectal cancer differentiation through the regulation of Skp2-p21/p27 axis. Oncogene. 2018;37(13):175974.

15. Tamura T, Ichikawa T, Nakahata S, Kondo Y, Tagawa Y, Yamamoto K, Nagai K, Baba T, Yamaguchi R, Futakuchi M, et al. Loss of NDRG2 Expression Confers Oral Squamous Cell Carcinoma with Enhanced Metastatic Potential. Cancer Res. 2017;77(9):2363-74.

16. Nakahata S, Ichikawa T, Maneesaay P, Saito Y, Nagai K, Tamura T, Manachai N, Yamakawa N, Hamasaki M, Kitabayashi I, et al. Loss of NDRG2 expression activates PI3K-AKT signalling via PTEN phosphorylation in ATLL and other cancers. Nat Commun. 2014;5:3393.

17. Yang $C L$, Zheng XL, Ye K, Ge H, Sun YN, Lu YF, Fan QX. NDRG2 suppresses proliferation, migration, invasion and epithelial-mesenchymal transition of esophageal cancer cells through regulating the AKT/XIAP signaling pathway. Int J Biochem Cell Biol. 2018;99:43-51.

18. Kim YJ, Yoon SY, Kim JT, Choi SC, Lim JS, Kim JH, Song EY, Lee HG, Choi I, Kim JW. NDRG2 suppresses cell proliferation through down-regulation of AP-1 activity in human colon carcinoma cells. Int J Cancer. 2009;124(1):7-15.

19. Hwang J, Kim Y, Kang HB, Jaroszewski L, Deacon AM, Lee H, Choi WC, Kim KJ, Kim CH, Kang BS, et al. Crystal structure of the human N-Myc downstream-regulated gene 2 protein provides insight into 
its role as a tumor suppressor. J Biol Chem. 2011;286(14):12450-60.

20. Shen L, Qu X, Ma Y, Zheng J, Chu D, Liu B, Li X, Wang M, Xu C, Liu N, et al. Tumor suppressor NDRG2 tips the balance of oncogenic TGF-beta via EMT inhibition in colorectal cancer. Oncogenesis. 2014;3:e86.

21. Pan T, Zhang M, Zhang F, Yan G, Ru Y, Wang Q, Zhang Y, Wei X, Xu X, Shen L, et al. NDRG2 overexpression suppresses hepatoma cells survival during metabolic stress through disturbing the activation of fatty acid oxidation. Biochem Biophys Res Commun. 2017;483(2):860-66.

22. Le A, Lane AN, Hamaker M, Bose S, Gouw A, Barbi J, Tsukamoto T, Rojas CJ, Slusher BS, Zhang H, et al. Glucose-independent glutamine metabolism via TCA cycling for proliferation and survival in $B$ cells. Cell Metab. 2012;15(1):110-21.

23. Inobe T, Matouschek A. Paradigms of protein degradation by the proteasome. Curr Opin Struct Biol. 2014;24:156-64.

24. Li X, Cheng KKY, Liu Z, Yang JK, Wang B, Jiang X, Zhou Y, Hallenborg P, Hoo RLC, Lam KSL, et al. The MDM2-p53-pyruvate carboxylase signalling axis couples mitochondrial metabolism to glucosestimulated insulin secretion in pancreatic beta-cells. Nat Commun. 2016;7:11740.

25. Wattanavanitchakorn S, Ansari IH, El Azzouny M, Longacre MJ, Stoker SW, MacDonald MJ, Jitrapakdee S. Differential contribution of pyruvate carboxylation to anaplerosis and cataplerosis during non-gluconeogenic and gluconeogenic conditions in HepG2 cells. Arch Biochem Biophys. 2019;676:108124.

26. Altman BJ, Stine ZE, Dang CV. From Krebs to clinic: glutamine metabolism to cancer therapy. Nat Rev Cancer. 2016;16(10):619-34.

27. Lao-On U, Rojvirat P, Chansongkrow P, Phannasil P, Siritutsoontorn S, Charoensawan V, Jitrapakdee S. c-Myc directly targets an over-expression of pyruvate carboxylase in highly invasive breast cancer. Biochim Biophys Acta Mol Basis Dis. 2020;1866(3):165656.

28. Qian X, Li X, Cai Q, Zhang C, Yu Q, Jiang Y, Lee JH, Hawke D, Wang Y, Xia Y, et al. Phosphoglycerate Kinase 1 Phosphorylates Beclin1 to Induce Autophagy. Mol Cell. 2017;65(5):917-31. e6.

29. Lie S, Wang T, Forbes B, Proud CG, Petersen J. The ability to utilise ammonia as nitrogen source is cell type specific and intricately linked to GDH, AMPK and mTORC1. Sci Rep. 2019;9(1):1461.

30. Jin HO, Hong SE, Kim JY, Jang SK, Kim YS, Sim JH, Oh AC, Kim H, Hong YJ, Lee JK, et al. Knockdown of PSAT1 Enhances Sensitivity of NSCLC Cells to Glutamine-limiting Conditions. Anticancer Res. 2019;39(12):6723-30.

31. Sun L, Song L, Wan Q, Wu G, Li X, Wang Y, Wang J, Liu Z, Zhong X, He X, et al. cMyc-mediated activation of serine biosynthesis pathway is critical for cancer progression under nutrient deprivation conditions. Cell Res. 2015;25(4):429-44.

32. Le Grand M, Mukha A, Puschel J, Valli E, Kamili A, Vittorio O, Dubrovska A, Kavallaris M. Interplay between $\mathrm{MycN}$ and c-Myc regulates radioresistance and cancer stem cell phenotype in neuroblastoma upon glutamine deprivation. Theranostics. 2020;10(14):6411-29. 
33. Chandler CS, Ballard FJ. Inhibition of pyruvate carboxylase degradation and total protein breakdown by lysosomotropic agents in 3T3-L1 cells. Biochem J. 1983;210(3):845-53.

34. Dombi E, Mortiboys H, Poulton J. Modulating Mitophagy in Mitochondrial Disease. Curr Med Chem. 2018;25(40):5597-612.

35. Balss J, Meyer J, Mueller W, Korshunov A, Hartmann C, von Deimling A. Analysis of the IDH1 codon 132 mutation in brain tumors. Acta Neuropathol. 2008;116(6):597-602.

\section{Figures}




\section{Figure 1}

A
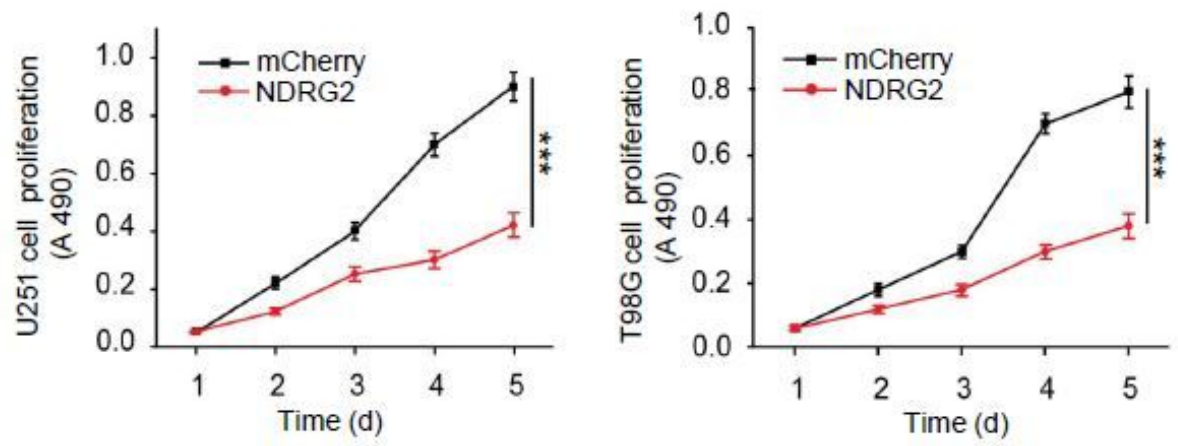

B

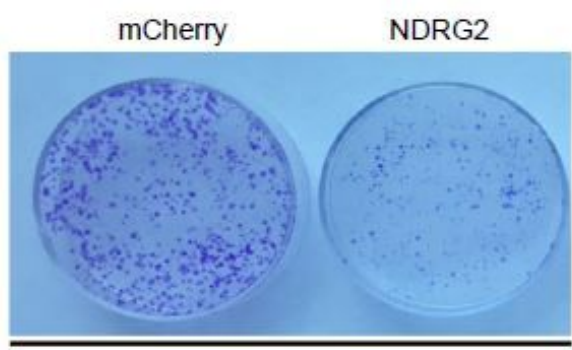

U251

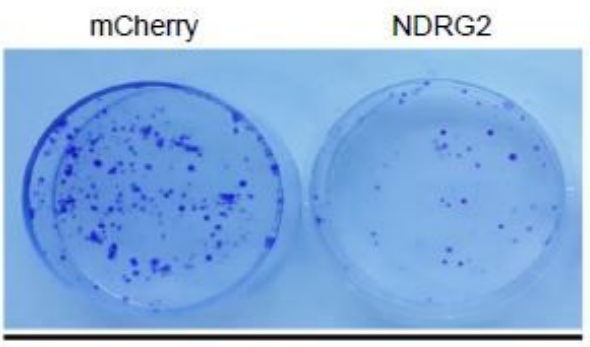

T98G

C

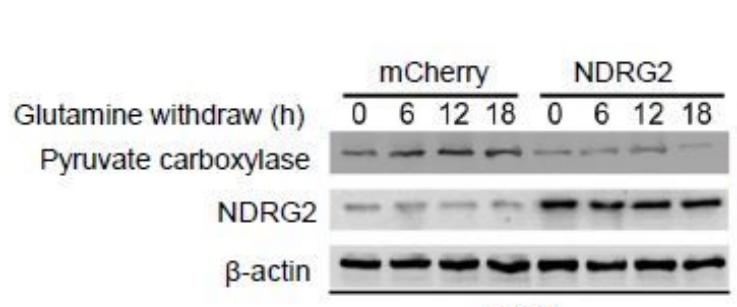

U251

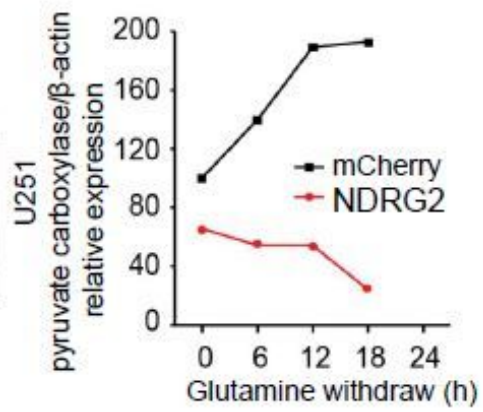

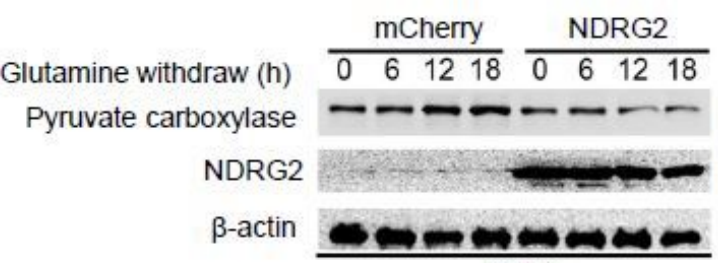

T98G

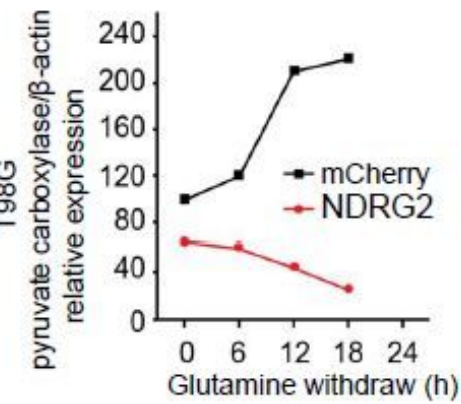

\section{Figure 1}

NDRG2 inhibits the proliferation and blocks the upregulation of pyruvate carboxylase expression in glutamine deficient glioma cells. A U251 and T98G cells were infected with lentivirus containing NDRG2 or mCherry, and cell viability was measured with MTT assays. B Equal numbers of NDRG2overexpressing U251 (T98G) cells and control cells were seeded onto $60 \mathrm{~mm}$ dishes. After 14 days, the cells were fixed and stained with crystal violet. C U251 and T98G cells were infected with NDRG2 or 
mCherry lentivirus for 48 hours, and then cultured in DMEM medium without glutamine for the indicated periods. The protein levels of pyruvate carboxylase and NDRG2 at different times were detected by Western blotting analysis. The relative protein levels of pyruvate carboxylase to $\beta$-actin were quantified by densitometry. All data shown are the mean \pm SEM of three independent experiments. ${ }^{* \star} p<0.001$

\section{Figure 2}

A
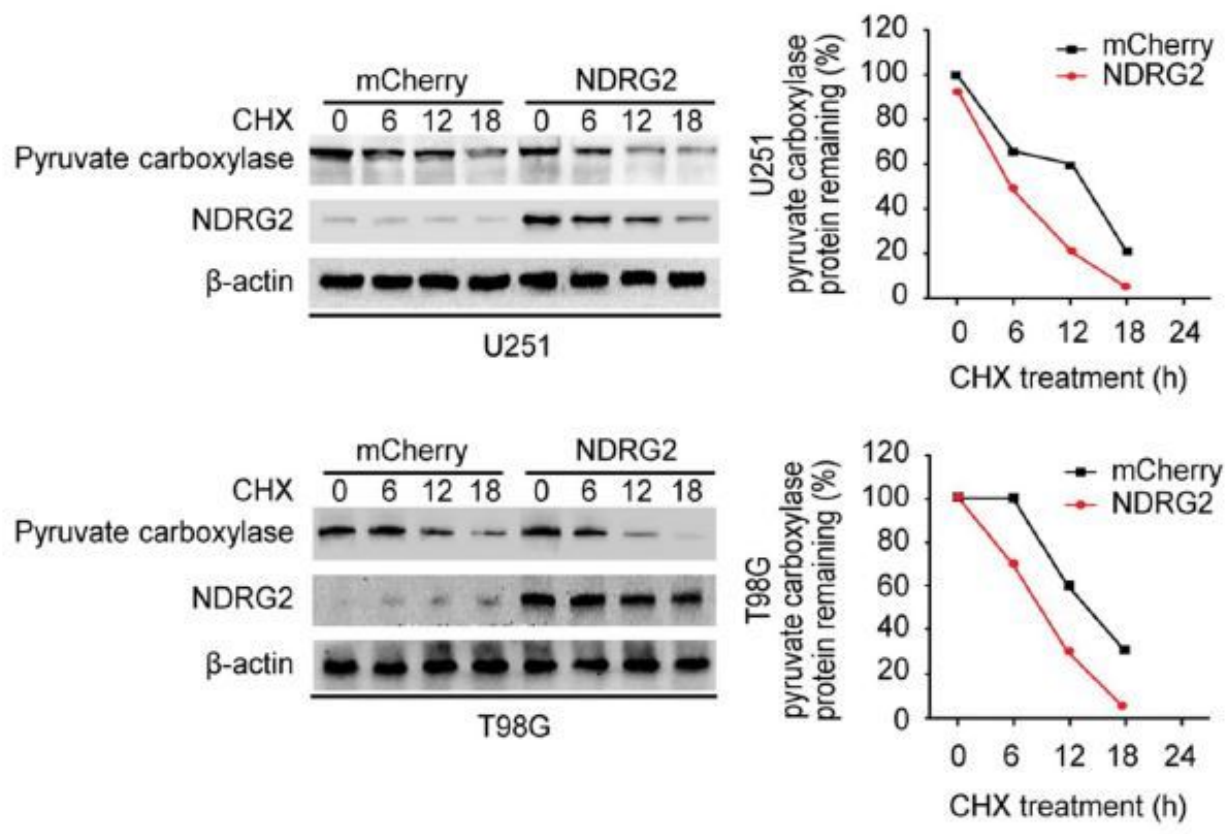

B

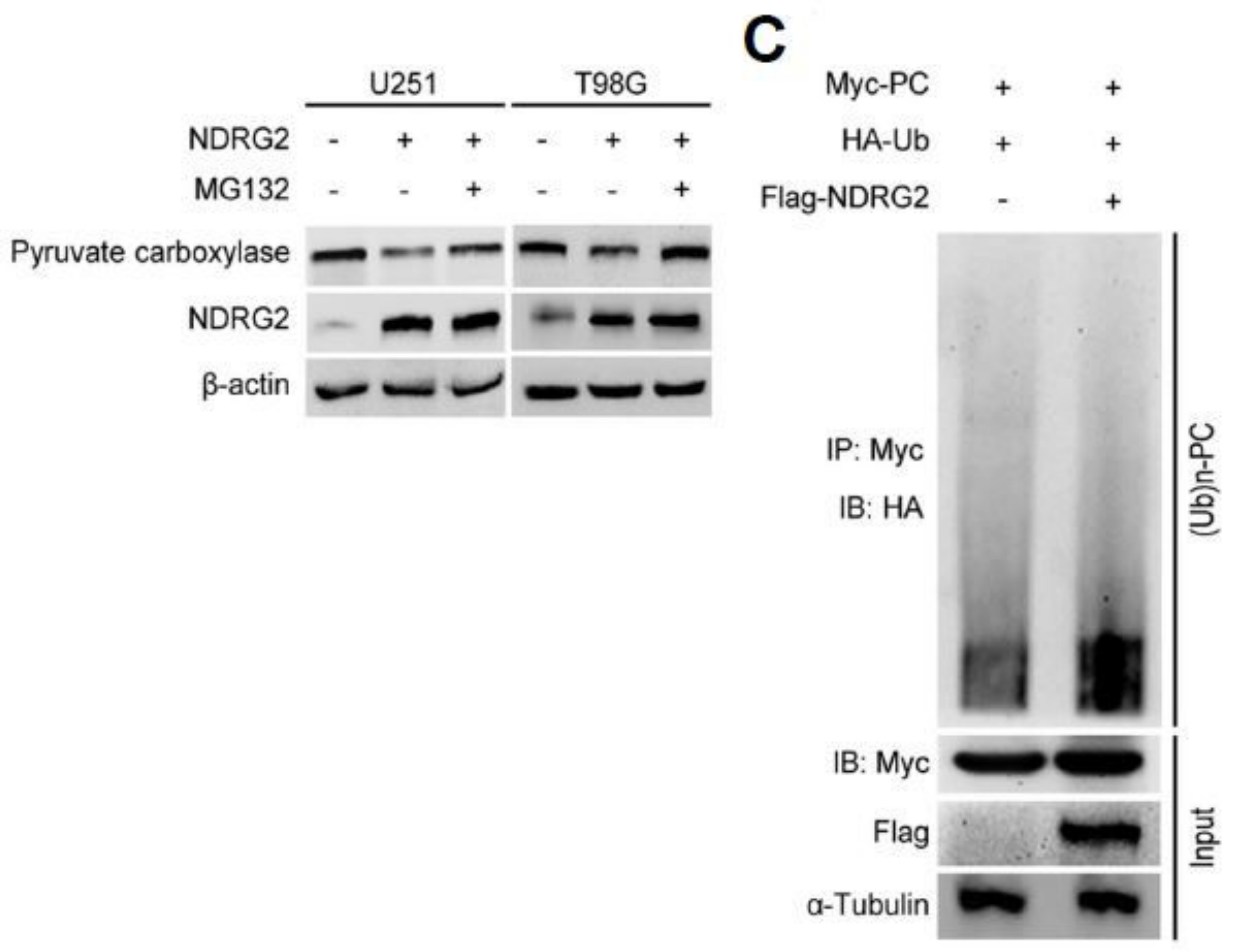

Figure 2 
NDRG2 induces the degradation of pyruvate carboxylase upon glutamine deprivation. A U251 and T98G cells were infected with NDRG2 or mCherry lentivirus for 48 hours, cultured in DMEM medium without glutamine and treated with $50 \mu \mathrm{m}$ cycloheximide for the indicated periods. The protein levels of pyruvate carboxylase and NDRG2 at different times were detected by Western blotting analysis. The relative protein levels of pyruvate carboxylase to $\beta$-actin were quantified by densitometry. B U251 and T98G cells infected with NDRG2 or mCherry lentivirus were cultured in DMEM medium without glutamine and treated with $20 \mu \mathrm{m}$ MG132 for 18 hours, and Western blotting analysis of the protein levels of pyruvate carboxylase and NDRG2 was performed. C Western blotting analysis of the products of the in vivo ubiquitination assay in HEK293T cells transfected with the indicated plasmids and cultured in DMEM medium without glutamine was performed

\section{Figure 3}

A

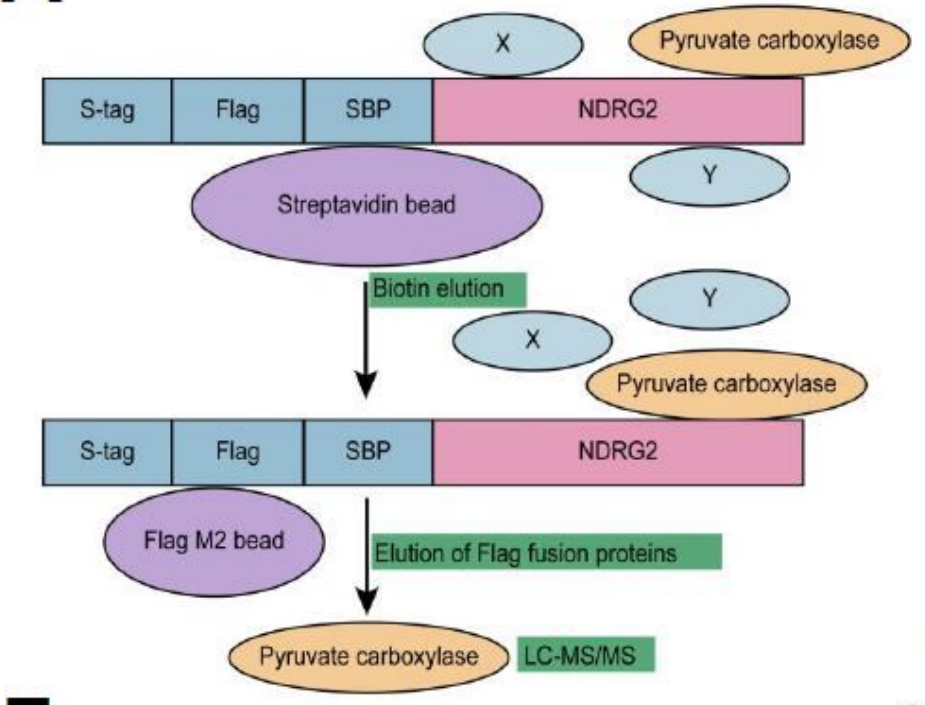

F
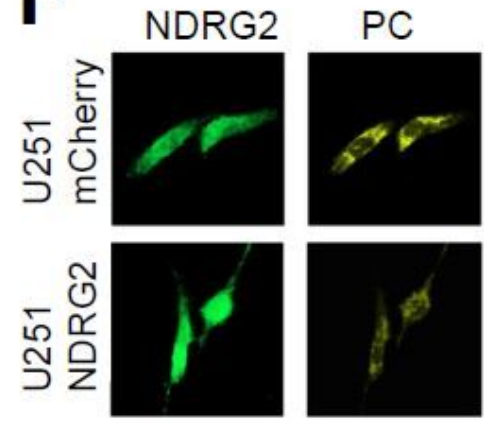

TAP Purification

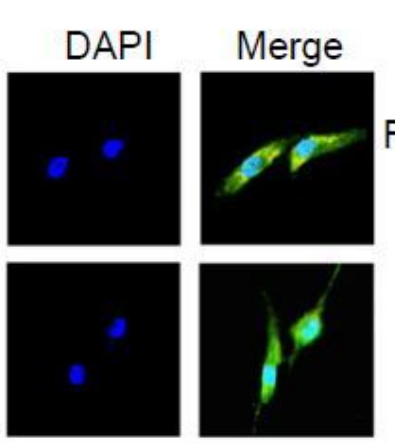

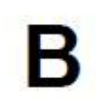

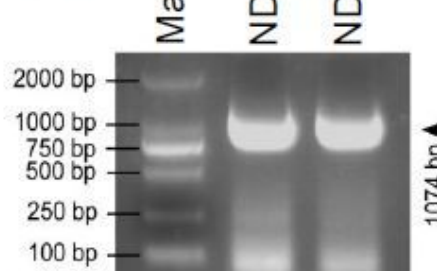

D

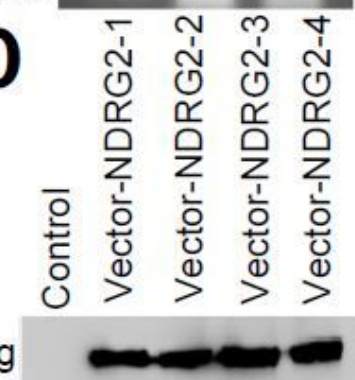

$\beta$-actin

Myc-PC Flag-NDRG2

IB:Myc
IB:Flag

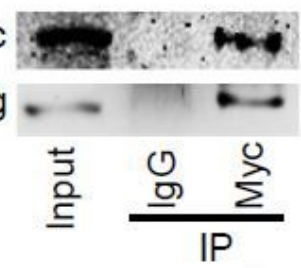

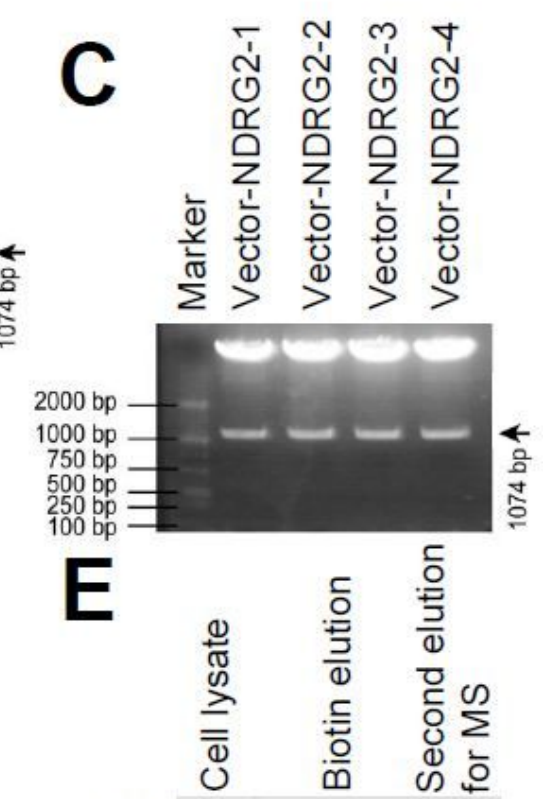

Flag

Myc-PC + + + Flag-NDRG2 +

IB:Myc

IB:Flag

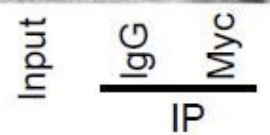

Figure 3

NDRG2 interacts with pyruvate carboxylase. A Graphic illustration of the tandem affinity and purification (TAP) procedure for mass spectrometry (TAP-MS). B PCR amplification of the NDRG2 gene from human astrocyte CDNA. C Digestion identification of recombinant plasmid S protein-Flag-Streptavidin binding protein tag-NDRG2 (SFB-NDRG2) with restriction enzyme by agarose gel electrophoresis. D HEK293T 
cells were transfected with the recombinant plasmid SFB-NDRG2, and Flag tag expression was detected by immunoblotting using an anti-Flag antibody. E Purified protein complexes for mass spectrometry were detected by immunoblotting using an anti-Flag antibody. F Subcellular localization of NDRG2 and pyruvate carboxylase in U251 cells. Immunocytochemical staining of U251 cells was performed using NDRG2 or PC antibody, and visualized using FITC- or Cy5-conjugated secondary antibody to show the localization of NDRG2 (green) and PC (yellow). DAPI staining (blue) reveals the cell nuclei. G Western blot analysis of ectopically expressed Flag-NDRG2 and Myc-PC reciprocally immunoprecipitated by anti-Myc in $293 \mathrm{~T}$ cells 


\section{Figure 4}

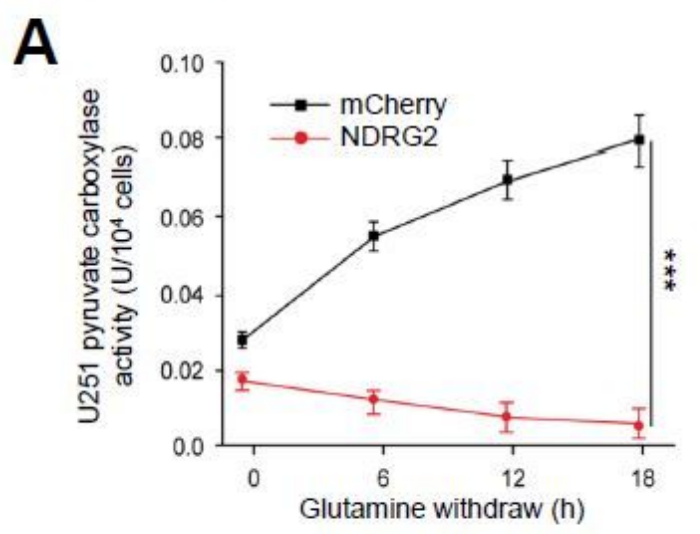

B
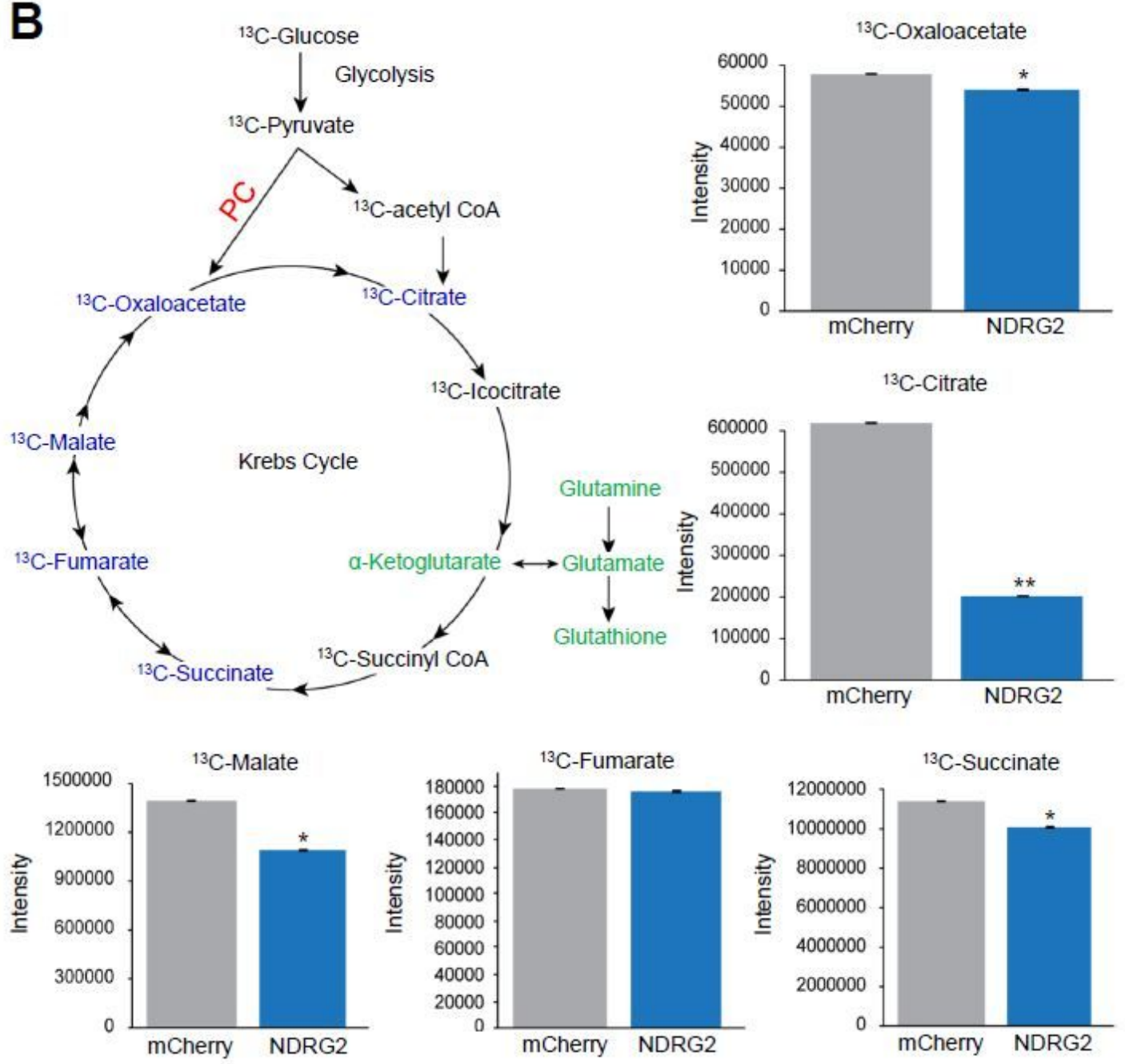

\section{Figure 4}

NDRG2 inhibits the activity of pyruvate carboxylase and glucose-dependent anaplerosis through pyruvate carboxylase in glioma cells. A U251 and T98G cells were infected with lentivirus containing NDRG2 or mCherry for 48 hours and incubated with DMEM medium without glutamine for the indicated times. The activity of pyruvate carboxylase in U251 and T98G cells was detected by a pyruvate carboxylase activity assay kit according to the manufacturer's recommendation. Total enzyme activity was normalized to the 
cell number. B U251 cells were infected with lentivirus containing NDRG2 or mCherry for 48 hours and incubated with DMEM medium containing 13C-labeled glucose without glutamine for 18 hours. The 13Clabeled organic acids in the TCA cycle were detected by LC-MS analysis. ${ }^{*} p<0.05,{ }^{* *} p<0.01,{ }^{* *} p<0.001$

\section{Figure 5}

A

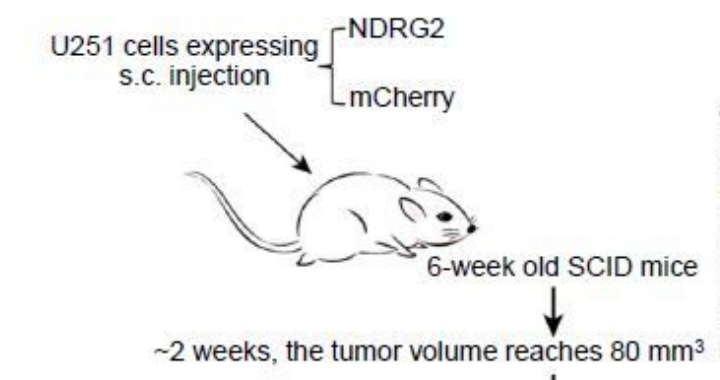

\begin{tabular}{llllllll}
0 & 3 & 7 & 10 & 14 & 18 & 21 & 24 (Days) \\
\hline$\uparrow$ & $\uparrow$ & $\uparrow$ & $\uparrow$ & $\uparrow$ & $\uparrow$ & $\uparrow$ & \\
i.p.drug administration & & &
\end{tabular}

C

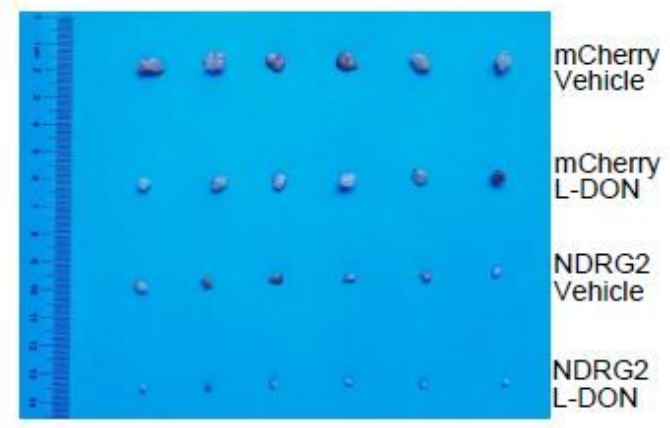

E

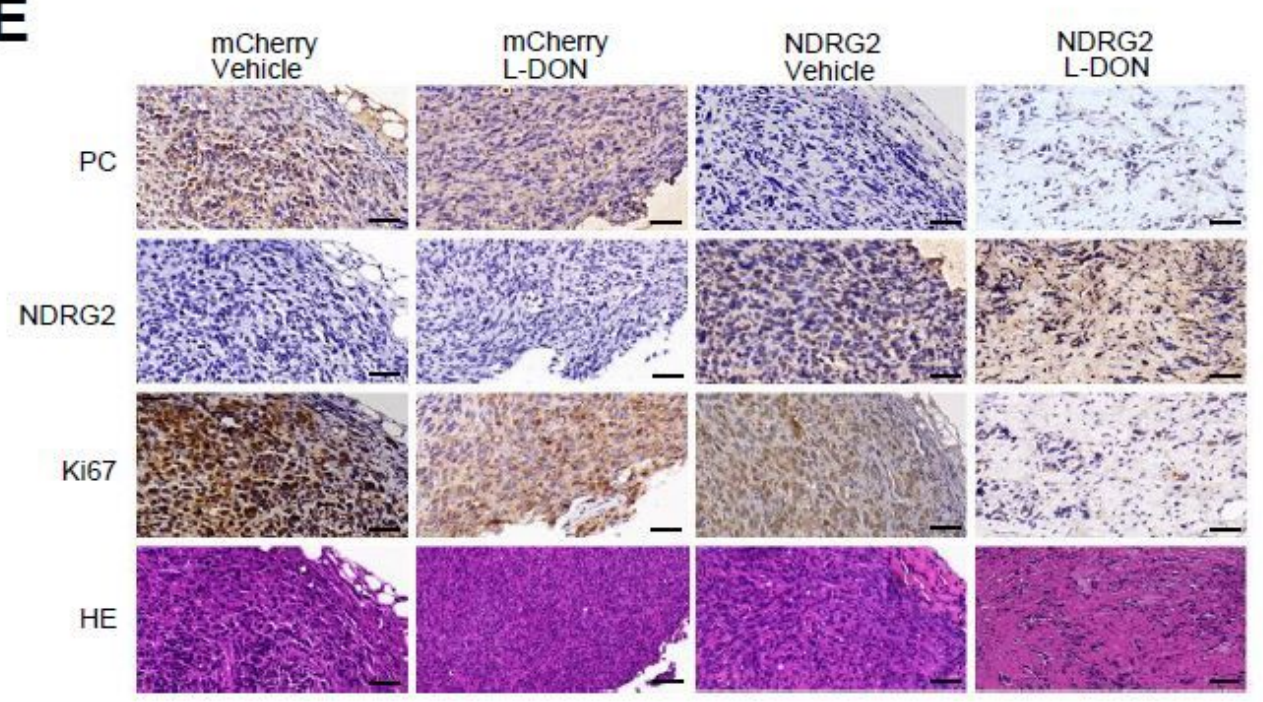

Figure 5

NDRG2 combined with glutamine antagonist inhibits the proliferation of glioma cells in vivo. A A schematic depicts the procedure of the establishment of NDRG2 overexpression xenograft models and 
inhibitor administration. Nude mice were injected subcutaneously with U251 cells that were infected with lentivirus containing NDRG2 or mCherry. Once the tumor size in nude mice of any group reached an average of $80 \mathrm{~mm} 3$, all mice were treated with $15 \mathrm{mg} / \mathrm{kg}$ L-DON or PBS 2 times per week for three consecutive weeks. The control mice received saline alone. B Tumor growth is shown. C Representative tumor formation was photographed after the mice were sacrificed. D Tumor weight was calculated at the end of the experiment. E Immunochemical staining with antibodies specific for PC, NDRG2 and Ki67 in xenografts. Scale bar, $50 \mu \mathrm{m}$. All data shown are the mean \pm SEM of three independent experiments. ${ }^{*} \mathrm{p}$ $<0.01, \star \star \star p<0.001$ 
Figure 6
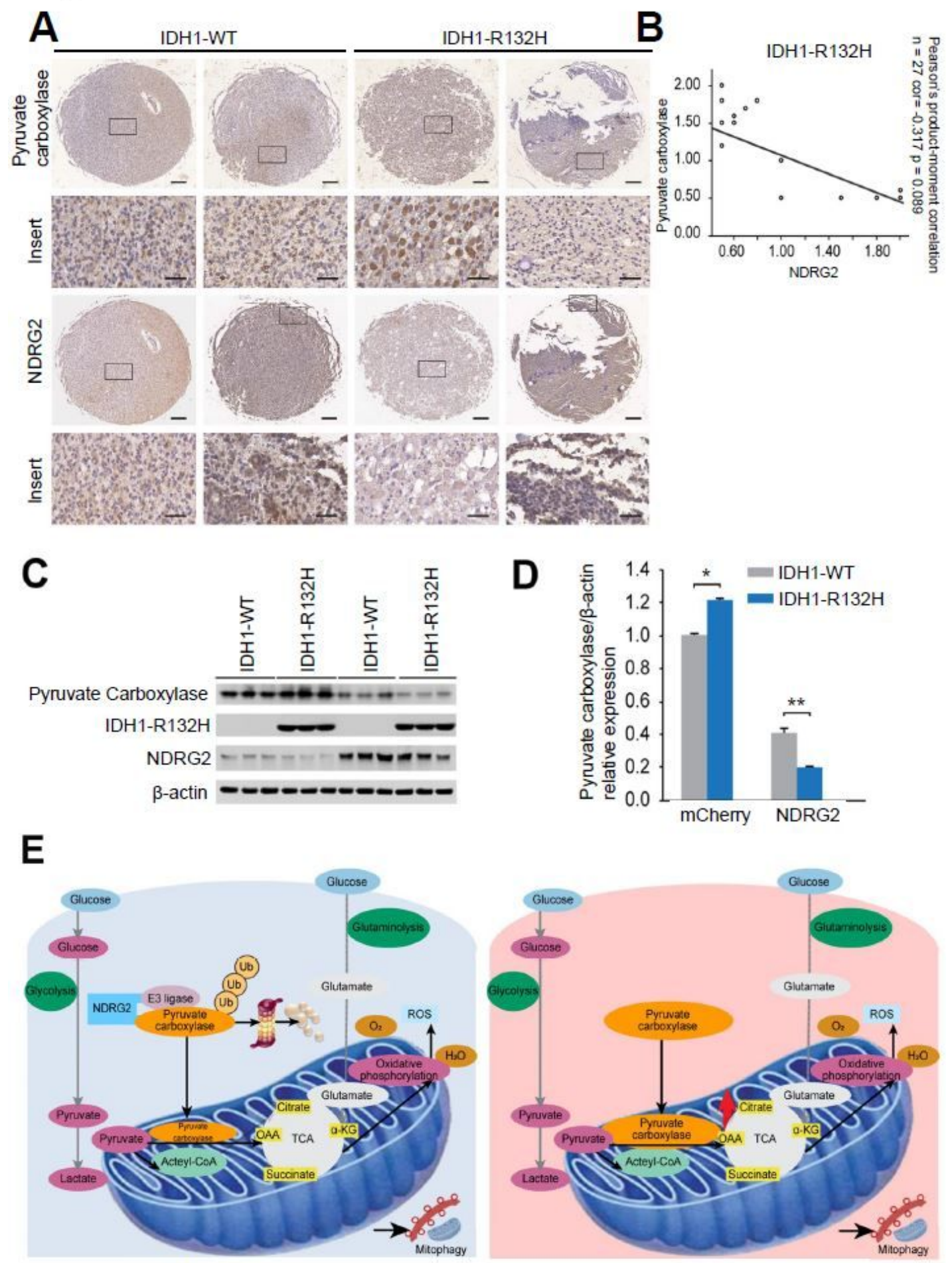

Homeostasis
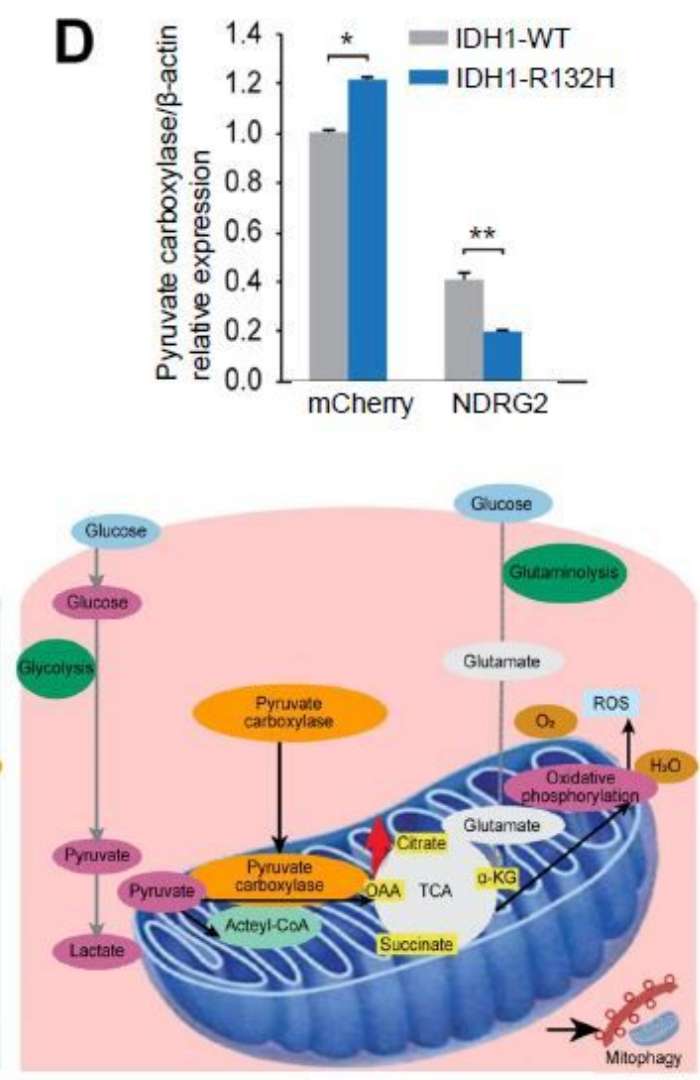

Glioma

\section{Figure 6}

The expression of NDRG2 had an inverse association with pyruvate carboxylase in IDH1(R132H)-mutant glioma cells. A NDRG2 and pyruvate carboxylase immunostaining of tissue microarrays comprising IDH1 wild-type and IDH1(R132H) glioma tissues with different differentiation states. Scale bar, $200 \mu \mathrm{m}$ and 50 $\mu \mathrm{m}$ (magnification). B Correlation analysis of the staining index for the expression of NDRG2 and PC proteins in the IDH1 wild-type glioma patient specimens $(n=27)$ and the IDH1-R132H mutant glioma 
patient specimens $(n=27)$. Pearson's product-moment correlation coefficients and the $P$ values are also shown. C NDRG2 and pyruvate carboxylase expression in the IDH1 wild-type and IDH1 (R132H) glioma U87 cells. D The relative protein levels of pyruvate carboxylase to $\beta$-actin were quantified by densitometry. E A schematic for the role of NDRG2 in regulating pyruvate carboxylase and pyruvate carboxylasemediated anaplerosis in glioma cells. In normal glial cells, NDRG2 promotes the degradation of pyruvate carboxylase before PC translocates into the mitochondria (left). NDRG2 loss leads to an increase in PCand PC-mediated anaplerosis under glutamine antagonism, and accelerates the tumorigenesis of glioma (right). ${ }^{\star} p<0.05,{ }^{*} \mathrm{p}<0.01$

\section{Supplementary Files}

This is a list of supplementary files associated with this preprint. Click to download.

- SuppFig1.jpg

- SuppFig2.jpg

- ProteinldentificationTable.xlsx

- SuppleTables.doc 